\title{
A Novel Approach to Increase the Goodness of Fits with an Application to Real and Simulated Data Sets
}

\author{
Muhammad Farooq, ${ }^{1}$ Qamruz zaman, ${ }^{1}$ Muhammad Ijaz ${ }^{D},{ }^{2}$ Said Farooq Shah, \\ and Mutua Kilai ${ }^{3}$ \\ ${ }^{1}$ Department of Statistics, University of Peshawar, Peshawar, Pakistan \\ ${ }^{2}$ Department of Statistics, The University of Haripur, Haripur, Pakistan \\ ${ }^{3}$ Department of Statistics, Jomo Kenyatta University of Agriculture and Technology, Juja, Kenya
}

Correspondence should be addressed to Muhammad Ijaz; ijaz.statistics@gmail.com

Received 25 May 2021; Accepted 24 July 2021; Published 2 August 2021

Academic Editor: Ishfaq Ahmad

Copyright (c) 2021 Muhammad Farooq et al. This is an open access article distributed under the Creative Commons Attribution License, which permits unrestricted use, distribution, and reproduction in any medium, provided the original work is properly cited.

\begin{abstract}
In practice, the data sets with extreme values are possible in many fields such as engineering, lifetime analysis, business, and economics. A lot of probability distributions are derived and presented to increase the model flexibility in the presence of such values. The current study also focuses on investigations to derive a new probability model New Flexible Family (NFF) of distributions. The significance of NFF is carried out using the Weibull distribution called New Flexible Weibull distribution or in short NFW. Various mathematical properties of NFW have been discussed including the estimation of parameters and entropy measures. Two real data sets with extreme values and a simulation study have been conducted so as to delineate the importance of NFW. Furthermore, NFW is compared with other existing probability distributions; numerically, it has been observed that the new mechanism of producing the lifetime probability distributions plays a significant role in making predictions about the population than others using the data sets with extreme values.
\end{abstract}

\section{Introduction and Problem Statement}

Due to wide applicability of the probability models in various disciplines, it remains a great interest of the researchers to increase the precision and validity of predictions and forecasting through probability functions in the presence of extreme values. For example, Ijaz et.al. [1] produced a new family of lifetime distributions and discussed its various statistical properties. The probability function of [1] can increase the reliability than others for the lifetime data analysis. Other prominent families of distributions include the Beta-G proposed by Eugene et al. [2], Jones [3], and Zografos and Balakrishnan [4] suggested a Gamma-G (type-1) family, Mc$\mathrm{G}$ proposed by Alexander et al. [5], Amini et al. [6] delineate the Log-Gamma-G type-2 family, Gamma-G (type-2) studied by Ristić and Balakrishnan [7], Torabi and Narges Montazeri [8] discussed Gamma-G (type-3), Weibull-X family of distributions introduced by Alzaatreh et al. [9], and exponentiated generalized class of Cordeiro et al. [10]. For a detailed discussion on the development of such new families of distributions, we refer to see [11-19].

The existing probability distributions have some limitations while modeling the lifetime data. First of all, these distributions are unable to model the nonmonotonic hazard function; for example, exponential distribution can only model the constant hazard rate function, while gamma distribution can only model the monotonically increasing failure rate function. Secondly, even if the existing probability functions model the data, they provide a bad fit to the real data. In practice, a lot of data sets follow a shape rather than the constant or monotonically increasing failure function. For instance, the lifetime of accident rate or an electronic device follows the pattern of nonmonotonic hazard rate function [1].

This paper presents another contribution to the existing theory of probability functions that will overcome the limitations of the existing distributions and some others recently developed. 


\section{Methodology to Proposed New Family of Distributions}

A lot of probability distributions are introduced by defining the new generator called families of probability distributions; for example, [1] introduced a Gull Power Alpha family of distribution, and for other families, we refer to $[6,9,15,20,21]$ and [22-29]. In this paper, a new family (NFF) is presented by introducing a new scale parameter "a" which is given by

$$
F(x)=\frac{a}{1-a}\left(a^{-F(y)}-1\right), \quad a \neq 1,
$$

where $F(y)$ is the CDF of the baseline distribution.

The probability density function of $F(x)$ is

$$
f(x)=\frac{a}{a-1}\left(\frac{\log (a) f(y)}{a^{F(y)}}\right), \quad a \neq 1 .
$$

\section{Special Form of the NFF}

This portion demonstrates the specific form of NFF by utilizing the CDF of the Weibull distribution called new Weibull distribution (NFW). The cumulative distribution function (CDF) of the Weibull distribution [30] is presented by

$$
F(y)=1-e^{-b y^{c}}, \quad y>0,
$$

where $b$ in the above equation represents scale parameter and $c$ represents the shape parameter.

By replacing (3) in (1), the CDF and PDF of NFW are, respectively, given by

$$
\begin{aligned}
& F(x)=\frac{a}{1-a}\left(a^{-\left(1-e^{-b x^{c}}\right)}-1\right), \quad a \neq 1, b, c>0, \\
& f(x)=\frac{a^{e^{-b x}} \ln (a) b c e^{-b x^{c}}}{a-1}, \quad a \neq 1, b, c>0 .
\end{aligned}
$$

Figure 1 presents numerous shapes of the CDF and PDF with distinct set of parameter values.

3.1. The Survival and Hazard Rate Function. The survival and hazard rate function of NFW is defined by

$S(x)=1-F(x)$, and using (4), we get

$$
=1-\left(\frac{a}{1-a}\left(a^{-\left(1-e^{-b x^{c}}\right)}-1\right)\right) \text {. }
$$

After simplification, the final result is given as follows:

$$
=\frac{a\left(a^{-e^{-b x^{c}}}-1\right)}{(1-a) a^{1-e^{-b x^{c}}} .}
$$

The hazard rate function of NFW is provided by

$$
h(x)=\frac{f(x)}{S(x)}
$$

Using (4) and (5), we finally obtained

$$
=\frac{\left(\left(a^{e^{-b x^{c}}} \ln (a) b c x^{c-1} e^{-b x^{c}}\right) /(a-1)\right)}{1-\left((a /(1-a))\left(a^{-\left(1-e^{-b x^{c}}\right)}-1\right)\right)} .
$$

Finally, the following result is obtained:

$$
h(x)=-\frac{\left(\ln (a) b c x^{c-1} e^{-b x^{c}}\right)}{\left(a^{-e^{-b x^{c}}}-1\right)} .
$$

Figure 2 demonstrates the nature of the hazard rate function for different values of parameter.

3.2. The Quantile Function and Median. The quantile function and median of NFW is defined by

$$
F(x)=p(X \leq x)=q,
$$

where $q$ is a standard uniform random variable.

Substituting (4), we obtain the result

$$
\frac{a}{1-a}\left(a^{-\left(1-e^{-b x^{c}}\right)}-1\right)=q \text {. }
$$
result:

The solution of equation (12) for $x$ will give the following

$$
x=\left(-\frac{1}{b} \log \left(\frac{\log (q(1-a))}{\log (a)}\right)\right)^{1 / c} .
$$

For median, consider $q=0.5$ in equation (13).

\section{The $\boldsymbol{R}^{\text {th }}$ Moments}

The $r^{\text {th }}$ moments about origin, suppose $u_{r}^{\prime}$ is defined as

$$
E\left(x^{r}\right)=\int_{0}^{\infty} x^{r} f(x) \mathrm{d} x .
$$

Recalling (5), we get

$$
=\int_{0}^{\infty} x^{r}\left(\frac{a^{e^{-b x^{c}}} \ln (a) b c x^{c-1} e^{-b x^{c}}}{a-1}\right) \mathrm{d} x .
$$

The simplified form of the above integral form is

$$
=\frac{\ln (a) b c}{a-1} \sum_{n=0}^{\infty} \frac{(\ln (a))^{n}}{n !} \int_{0}^{\infty} x^{r} x^{c-1} e^{-(n+1) b x^{c}} \mathrm{~d} x .
$$

Let $b x^{c}=z$, then $x=(z / b)^{1 / c}$, and $\mathrm{d} z=b c x^{c-1} \mathrm{~d} x$.

By using the above substitution in (16), finally, we get

$$
E\left(x^{r}\right)=\frac{\ln (a)}{(a-1)} \sum_{n=0}^{\infty} \frac{(\ln (a))^{n}}{n ! b^{r / c}}\left(\frac{(r+c) / c}{(n+1)^{(r+c) / c}}\right), \quad a \neq 1 .
$$

\section{Order Statistics}

Let $X_{1}, X_{2}, X_{3}, \ldots, X_{n}$ be ordered random variables from NFW, then the PDF of the HQIC order statistic is given by 

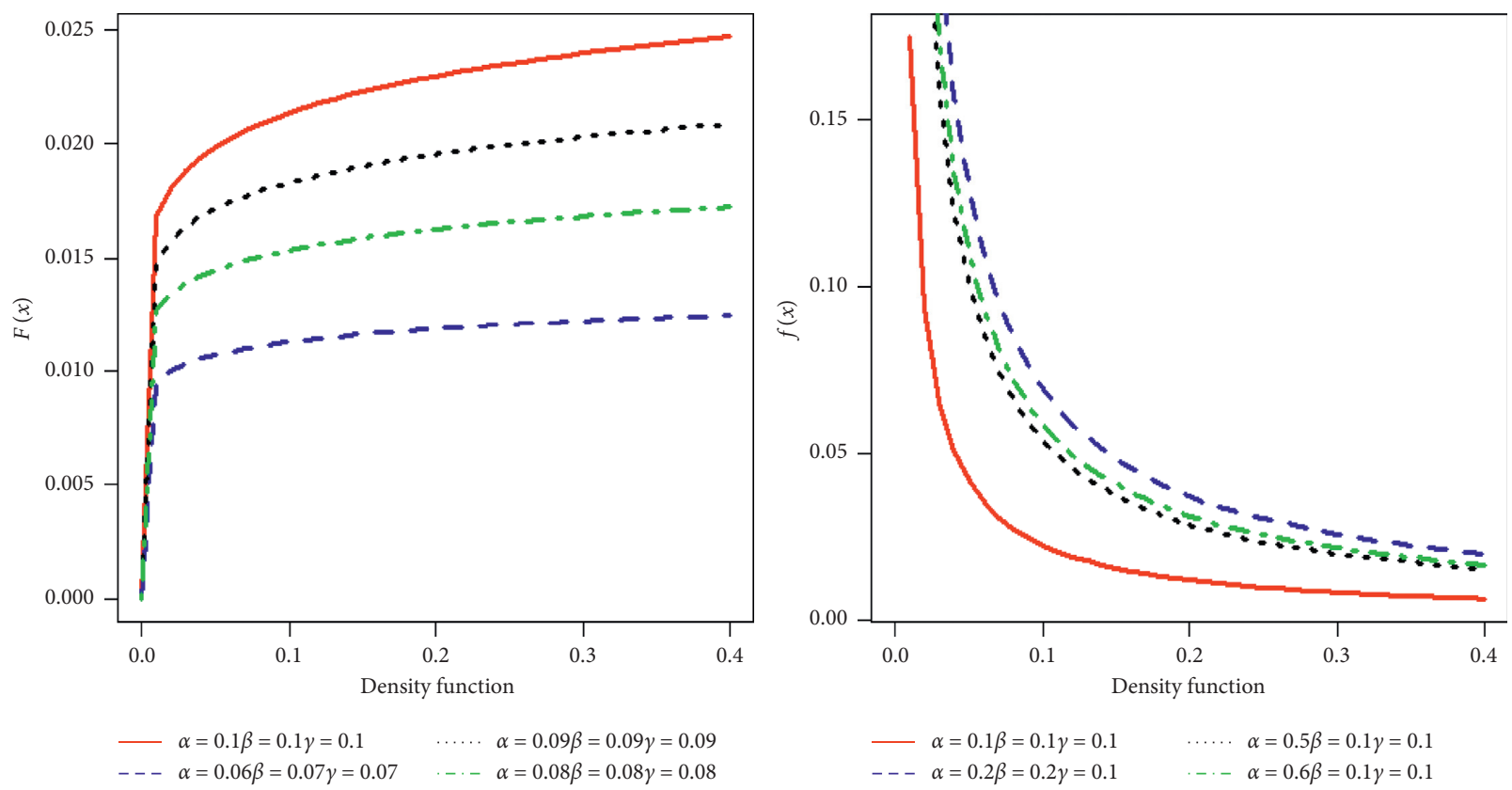

Figure 1: Plots of the CDF and PDF of NFW.

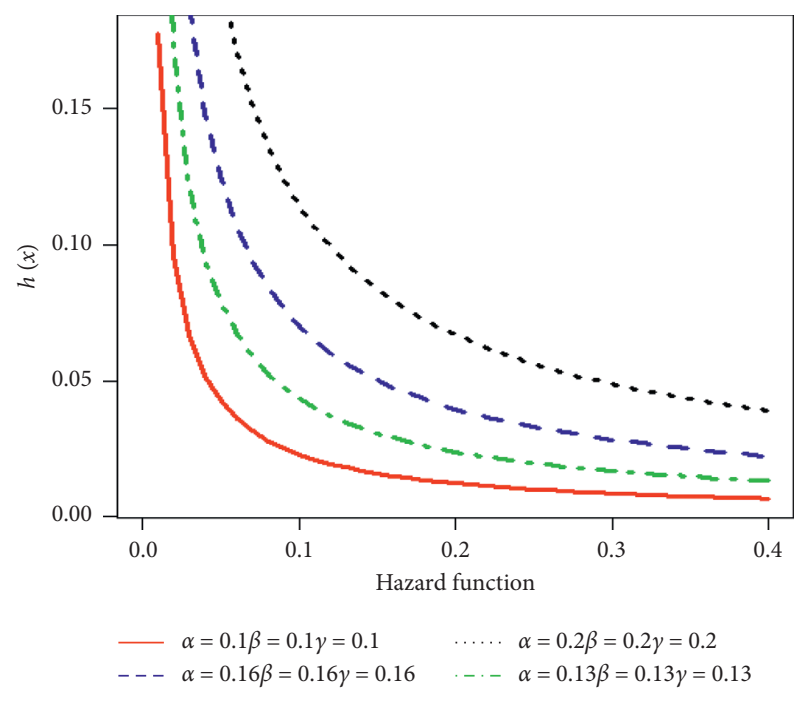

FIGURE 2: Plots of the hazard rate function of NFW.

$f_{i: n}(x)=\frac{n !}{(i-1) !(n-i) !} f(x) F(x)^{i-1}(1-F(x))^{n-i}$.

Using (4) and (5), the smallest and largest order statistic of NFW is defined by

$$
f_{1: n}(x)=n\left(\frac{a^{e^{-b x^{c}}} \log (a) b c x^{c-1} e^{-b x^{c}}}{a-1}\right)\left(\frac{1-a^{\left(e^{-b x^{c}}\right)}}{(1-a)}\right)^{n-1},
$$

and the largest order statistic is

$$
\begin{aligned}
f_{n, n}(x) & =n\left(\frac{a^{e^{-b x^{c}}} \log (a) b c x^{c-1} e^{-b x^{c}}}{a-1}\right) \\
& \cdot\left(\frac{a}{(1-a)}\left(\frac{\left.1-a^{\left(1-e^{-b x^{c}}\right.}\right)}{a^{\left(1-e^{-b x^{c}}\right)}}\right)\right)^{n-1} .
\end{aligned}
$$

\section{Parameter Estimation}

To increase the efficiency of the probability models, an estimation of the parameters also plays a vital role. Since the maximum likelihood estimation (MLE) method provides the most reliable estimates, therefore, for estimation of the parameters of NFW, we considered the MLE approach. A comprehensive discussion on maximum likelihood estimation was given by [31-33]. The likelihood function of (4) is defined by

$$
L=\frac{a^{e^{-\sum b x^{c}}} \ln ^{n}(a) b^{n} c^{n} \sum x^{c-1} e^{-b \sum x^{c}}}{a^{n}-1} .
$$

By taking the log of the above function, we get

$$
\begin{aligned}
\log (L)= & e^{-b \sum x^{c}} \log (a)+n \log (a)+n \log (b) \\
& +n \log (c)+(c-1) \sum \log (x)-b \sum x^{c}-n \log (a) .
\end{aligned}
$$

To obtain the estimate of the parameters, partial derivative with respect to various parameters $(a, b, c)$ was taken and equated the result to zero: 


$$
\begin{aligned}
\frac{\mathrm{d}}{\mathrm{d} a} \log (L)= & \frac{e^{-b \sum x^{c}}}{a}, \\
\frac{\mathrm{d}}{\mathrm{d} b} \log (L)= & -\sum x^{c} e^{-b \sum x^{c}} \log (a) \\
& +\frac{n}{b}-\sum x^{c}, \\
\frac{\mathrm{d}}{\mathrm{d} c} \log (L)= & -b c \log (a) e^{-b \sum x^{c}} \sum x^{c-1} \\
& +\frac{n}{c}+\sum \log (x)-b c \sum x^{c-1} .
\end{aligned}
$$

The exact values of the parameters are not possible as equations (23) to (25) are not in closed form. Still, one can use the numerical methods to get the ML estimates.

6.1. Asymptotic Confidence Bounds. The asymptotic confidence bounds for the parameters of $\operatorname{NFW}(a, b, c)$ are derived based on asymptotic distribution. To derive the required asymptotic confidence bounds, we are required the second time derivatives of (23)-(25):

$$
\begin{aligned}
& \frac{\mathrm{d}^{2}}{\mathrm{~d} a^{2}} \log (L)=-\frac{e^{-b \sum x^{c}}}{a^{2}} \\
& \frac{\mathrm{d}^{2}}{\mathrm{~d} a \mathrm{~d} b} \log (L)=\frac{-\sum x^{c} e^{-b \sum x^{c}}}{a}, \\
& \frac{\mathrm{d}^{2}}{\mathrm{~d} a \mathrm{~d} c} \log (L)=\frac{-b c \sum x^{c-1} e^{-b \sum x^{c}}}{a}, \\
& \frac{\mathrm{d}^{2}}{\mathrm{~d} b^{2}} \log (L)=\left(\sum x^{c}\right)^{2} e^{-b \sum x^{c}}-\frac{n}{b^{2}}, \\
& \frac{\mathrm{d}^{2}}{\mathrm{~d} b \mathrm{~d} c} \log (L)=-c \sum x^{c-1} e^{-b \sum x^{c}} \log (a) \\
& +b c \log (a) e^{-b \sum x^{c}} \sum x^{2 c-1}-c \sum x^{c-1} \\
& \frac{\mathrm{d}^{2}}{\mathrm{~d} c^{2}} \log (L)=-b \log (a) e^{-b \sum x^{c}} \sum x^{c-1} \\
& +b^{2} c \log (a) e^{-b \sum x^{2}} \sum x^{c} \log (c) \sum x^{c-1} \\
& -b c \log (a) e^{-b \sum x^{c}} \sum x^{c} \log (c)- \\
& \frac{n}{c^{2}} \quad-b \sum x^{c-1}-b c \sum x^{c-1} \log (c-1), \\
& \frac{\mathrm{d}^{3}}{\mathrm{~d} a \mathrm{~d} b \mathrm{~d} c} \log (L)=\frac{-c \sum x^{c-1} e^{-b \sum x^{c}}}{a}+\frac{b c e^{-b \sum x^{c}} \sum x^{2 c-1}}{a} .
\end{aligned}
$$

\section{Renyi Entropy}

The Renyi entropy of NFW is defined by

$$
R_{H}(x)=\frac{1}{1-p} \log \int_{0}^{\infty} f^{p}(x) \mathrm{d} x .
$$

Using (4), we get

$$
=\frac{1}{1-p} \log \int_{0}^{\infty}\left(\frac{a^{e^{-b x^{c}}} \ln (a) b c x^{c-1} e^{-b x^{c}}}{a-1}\right)^{p} \mathrm{~d} x \text {. }
$$

The more simplified form is

$$
=\left(\sum_{n=0}^{\infty} \frac{\log ^{n}(a)}{n !}\right)^{p} \int_{0}^{\infty} e^{-p(n+1) b x^{c}} x^{p(c-1)} \mathrm{d} x .
$$

Let $p(n+1) b x^{c}=z$, then $x=(z /(p(n+1) b))^{1 / c}$, and $\mathrm{d} x=\mathrm{d} z / p(n+1) b c x^{c-1}$.

By substituting the above result in (29), we obtained

$$
\begin{aligned}
& =\left(\sum_{n=0}^{\infty} \frac{\log ^{n}(a)}{n !}\right)^{p} \frac{1}{c(p(n+1) b)^{(p(c-1)-c+1+c) / c}} \int_{0}^{\infty} e^{-z} z^{((p(c-1)-c+1+c) / c)-1} \mathrm{~d} z \\
& =\left(\sum_{n=0}^{\infty} \frac{\log ^{n}(a)}{n !}\right)^{p} \frac{1}{c(p(n+1) b)^{(p(c-1)-c+1+c) / c}} \frac{p(c-1)+1}{c} .
\end{aligned}
$$

Finally, we obtained the result as

$$
\begin{aligned}
R_{H}(x)= & \frac{1}{1-p} \log \left(\sum_{n=0}^{\infty} \frac{\log ^{n}(a)}{n !}\right)^{p} \\
& \cdot \frac{1}{c(p(n+1) b)^{(p(c-1)-c+1+c) / c}} \frac{p(c-1)+1}{c} .
\end{aligned}
$$

\section{Mode}

The mathematical form of the NFW for the mode can be illustrated as follows:

$$
f^{\prime}(x)=0 .
$$

Using equation (4), we have

$$
\frac{\ln (a) b c}{a-1} \frac{\mathrm{d}}{\mathrm{d} y}\left[a^{e^{-b y^{c}}} y^{c-1} e^{-b y^{c}}\right]=0 .
$$

After simplifying the above expression, the result may be written as

$$
\left(b c y^{c}-c+1\right) e^{b y^{c}}+\ln (a) b c y^{c}=0 .
$$

The above function is an implicit function. It can be solved numerically under some restriction on the parameter values. 


\section{Skewness and Kurtosis}

The general functions for calculating the skewness $(S)$ and kurtosis $(K)$ of the NFW are given as follows:

$$
\begin{aligned}
& S=\frac{Q(6 / 8)+Q(2 / 8)-2 Q(4 / 8)}{Q(6 / 8)-Q(2 / 8)}, \\
& K=\frac{Q(7 / 8)+Q(3 / 8)-Q(5 / 8)-Q(1 / 8)}{Q(6 / 8)-Q(2 / 8)} .
\end{aligned}
$$

Table 1 depicts the skewness and kurtosis for different values of the parameters.

\section{Special Cases}

Following are the submodels of NFW.

10.1. Case $c=1$. By putting $c=1$ in (4) and (5), we get exactly the new probability distribution with the baseline CDF of the exponential distribution called new exponential distribution (NFE). The mathematical forms of the CDF and PDF are, respectively, given by

$$
\begin{aligned}
& F(x)=\frac{a}{1-a}\left(a^{-\left(1-e^{-b^{x}}\right)}-1\right), \quad a \neq 1, b>0, \\
& f(x)=\frac{a^{e^{-b x}} \ln (a) b c e^{-b x}}{a-1}, \quad a \neq 1, b>0 .
\end{aligned}
$$

10.2. Case $c=2$. By replacing $c=2$ in (4), and (5), we get exactly the new probability distribution with the baseline CDF of the Rayleigh distribution called new Rayleigh
TABLE 1: Numerical depiction of skewness and kurtosis.

\begin{tabular}{lcccc}
\hline$A$ & $B$ & $c$ & Skewness & Kurtosis \\
\hline 0.5 & 5.5 & 0.2 & -0.9382459 & 7.423786 \\
0.5 & 3.5 & 1.2 & -0.2016131 & 1.254566 \\
0.5 & 5 & 0.4 & -0.6793921 & 2.446662 \\
0.1 & 7 & 0.4 & -0.3588356 & 1.520896 \\
0.1 & 7 & 0.8 & -0.2783669 & 1.337107 \\
0.1 & 6 & 0.6 & -0.3055946 & 1.392746 \\
0.2 & 6 & 0.4 & -0.3963587 & 1.61144 \\
0.2 & 7 & 0.2 & -0.5893137 & 2.475911 \\
0.2 & 8 & 0.1 & -0.8363627 & 6.395 \\
0.1 & 10 & 0.4 & -0.3588356 & 1.520896 \\
\hline
\end{tabular}

distribution (NFR). The mathematical forms of the CDF and $\mathrm{PDF}$ are, respectively, given by

$$
\begin{aligned}
& F(x)=\frac{a}{1-a}\left(a^{-\left(1-e^{-b x^{2}}\right)}-1\right), \\
& f(x)=\frac{a^{e^{-b x^{2}}} \ln (a) b c x e^{-b x^{2}}}{a-1} .
\end{aligned}
$$

\section{Applications}

To check the performance of the proposed model, two real data sets with extreme values are considered, one consisting of nonmonotonic and the other monotonic hazard rate shapes. Various goodness of fit measures such as the Akaike information criteria (AIC), Hannan and Quinn information criteria (HQIC), Anderson darling $(A)$, Cramer-von Mises $(W)$, consistent Akaike information criteria (CAIC), and Bayesian information criteria (BIC) were considered to check the efficiency of the proposed model. Mathematically, these criteria are defined as

$$
\begin{aligned}
A & =-n-\frac{1}{n} \sum_{i=1}^{n}(2 i-1)\left[\log F\left(X_{i}\right)+\log \left(1-F\left(X_{n-i+1}\right)\right)\right]\left[\log F\left(X_{i}\right)+\log \left(1-F\left(X_{n-i+1}\right)\right)\right], \\
W & =\sum_{i=1}^{n}\left[F\left(X_{i}\right)-\frac{2 i-1}{2 n}\right]^{2}+\frac{1}{12 n}, \mathrm{AIC}=-2 L+2 p, \mathrm{AICc}=\mathrm{AIC}+\frac{2 p(p+1)}{n-p-1}, \\
\mathrm{CAIC} & =-2 L+P\{\log (n)+1\}, \quad \mathrm{BIC}=P \log (n)-2 L, \quad \mathrm{HQIC}=-2 L+2 P \log \{\log (n)\},
\end{aligned}
$$

where $L=L\left(\widehat{\psi} ; x_{i}\right)$ is the maximized likelihood function and $x_{i}$ is the random sample, $\widehat{\psi}$ is the MLE, and $p$ represents the number of parameters involved in the model.

Generally, the probability model that has the smallest number among these various criteria is judged as the best fitted model.
11.1. Data Set 1: Remission Time of Bladder Cancer Patients. The following data set represents the remission time of 128 bladder cancer patients taken from Aldeni and Famoye [20]. The values of the data set are given as follows:

$0.080,0.200,0.400,0.500,0.510,0.810,0.900,1.050$, $1.190,1.260,1.350,1.400,1.460,1.760,2.020,2.020,2.070$, 


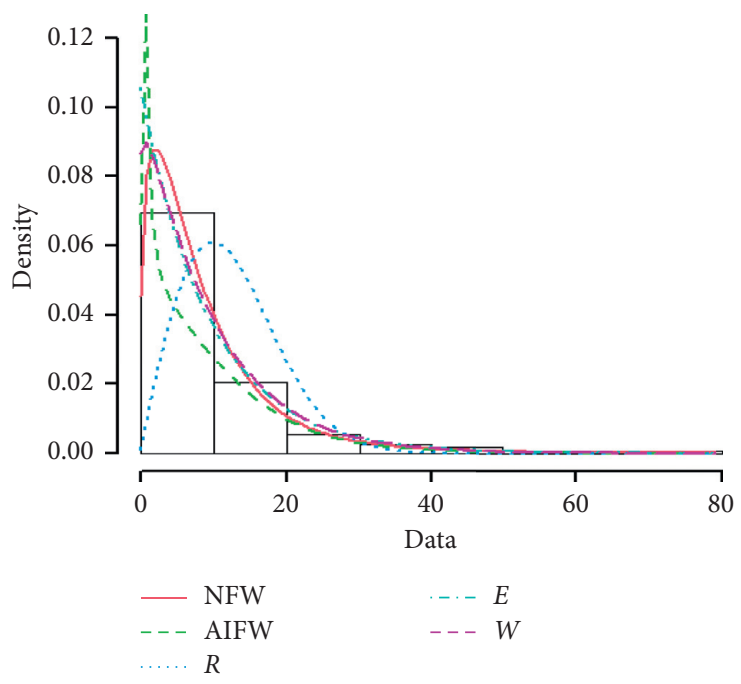

(a)

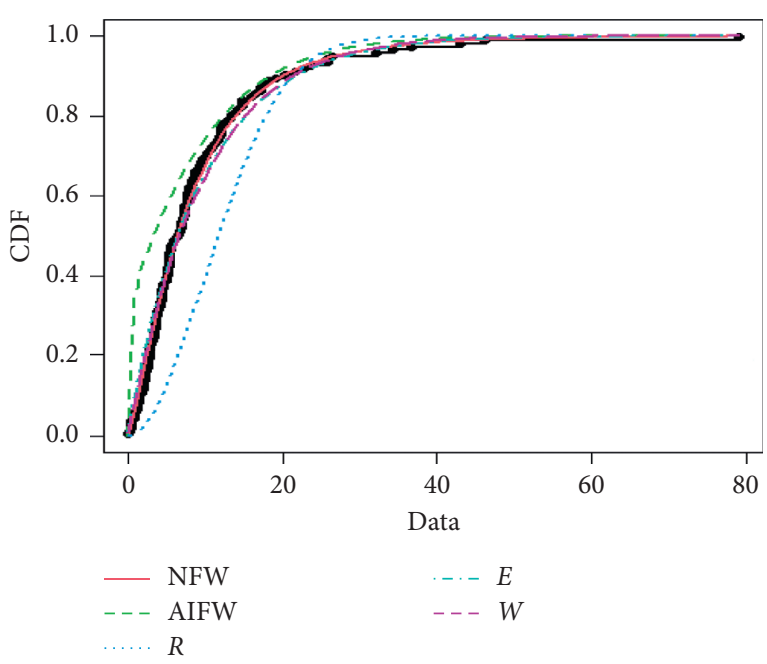

(b)

Figure 3: Theoretical and empirical PDF and CDF of NFW. (a) Histogram and theoretical densities. (b) Empirical and theoretical CDFs.

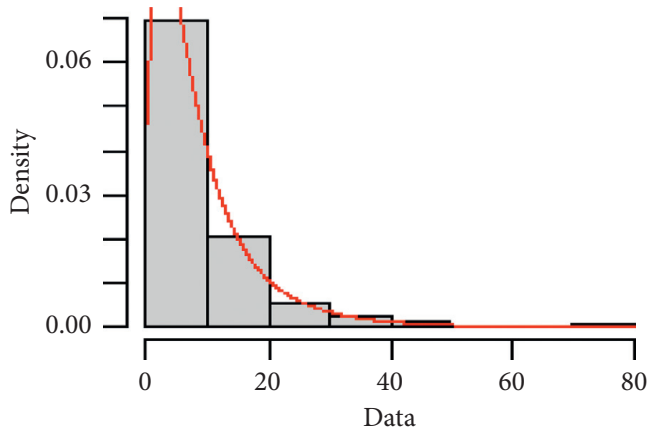

(a)

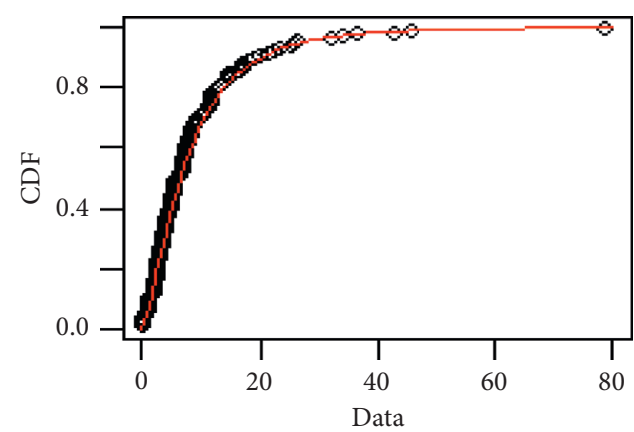

(c)

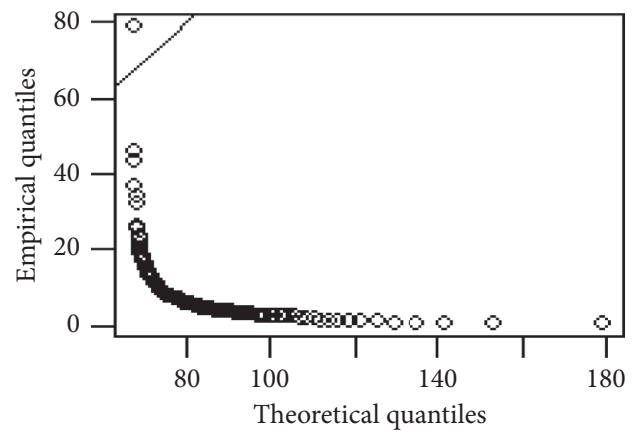

(b)

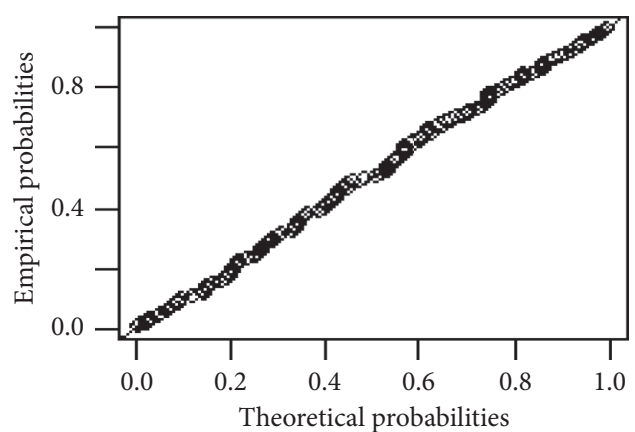

(d)

FIgure 4: Theoretical and empirical PDF and CDF with Q-Q plot and P-P plot. (a) Empirical and theoretical densities. (b) Q-Q plot. (c) Empirical and theoretical CDFs. (d) P-P plot.

$2.090,2.230,2.260,2.460,2.540,2.620,2.640,2.690,2.690$, $2.750,2.830,2.870,3.020,3.250,3.310,3.360,3.360,3.480$, $3.520,3.570,3.640,3.700,3.820,3.880,4.180,4.230,4.260$, $4.330,4.340,4.400,4.500,4.510,4.870,4.980,5.060,5.090$, $5.170,5.320,5.320,5.340,5.410,5.410,5.490,5.620,5.710$,
$5.850,6.250,6.540,6.760,6.930,6.940,6.970,7.090,7.260$, 7.280, 7.320, 7.390, 7.590, 7.620, 7.630, 7.660, 7.870, 7.930, $8.260,8.370,8.530,8.650,8.660,9.020,9.220,9.470,9.740$, $10.06,10.34,10.66,10.75,11.25,11.64,11.79,11.98,12.02$, $12.03,12.07,12.63,13.11,13.29,13.80,14.24,14.76,14.77$, 


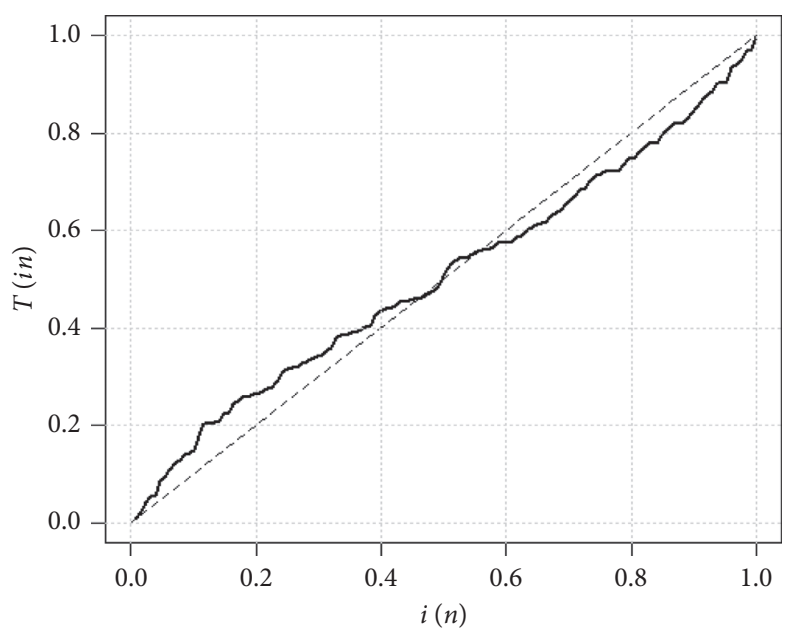

Figure 5: TTT plot of remission time data.

TABLe 2: Maximum likelihood estimates and their standard errors.

\begin{tabular}{|c|c|c|c|c|c|}
\hline Model & $W$ & $A$ & MLE & Standard error & $\begin{array}{c}-\log \\
\text { (likelihood) }\end{array}$ \\
\hline New Flexible Weibull & 0.05380675 & 0.333476 & $\begin{array}{c}10.165737860 .03040949 \\
1.22621069\end{array}$ & $\begin{array}{c}8.776131690 .01225008 \\
0.08579209\end{array}$ & 411.0722 \\
\hline Weibull exponential & 0.2145276 & 1.282891 & $\begin{array}{c}3.958105050 .01796843 \\
0.85819193\end{array}$ & $\begin{array}{c}1.2140895810 .004666546 \\
0.059280045\end{array}$ & 419.8998 \\
\hline Weibull & 0.1308177 & 0.7832353 & 0.094382921 .04576466 & 0.019126240 .06742473 & 414.0874 \\
\hline Exponential & 0.1192893 & 0.7159703 & 0.1067695 & 0.009436355 & 414.3419 \\
\hline Rayleigh & 0.4669078 & 2.732901 & 0.005079773 & 0.0004307331 & 491.2659 \\
\hline $\begin{array}{l}\text { Algoharai inverse flexible } \\
\text { Weibull }\end{array}$ & 0.5735798 & 3.457475 & 0.16774040 .1231948 & 0.025087750 .01045528 & 451.0704 \\
\hline
\end{tabular}

TABLE 3: Goodness of fit measures of the NFW for remission time data.

\begin{tabular}{|c|c|c|c|c|}
\hline Models & AIC & CAIC & $\mathrm{BIC}$ & HQIC \\
\hline New Flexible Weibull & 828.1444 & 828.3379 & 836.7005 & 831.6207 \\
\hline Weibull exponential & 845.7996 & 845.9931 & 854.3557 & 849.276 \\
\hline Weibull & 832.1747 & 832.2707 & 837.8788 & 834.4923 \\
\hline Exponential & 830.6838 & 830.7155 & 833.5358 & 832.8426 \\
\hline Rayleigh & 984.5318 & 984.5635 & 987.3838 & 985.6906 \\
\hline Algoharai inverse flexible Weibull & 906.1409 & 906.2369 & 911.8449 & 908.4585 \\
\hline
\end{tabular}

$14.83,15.96,16.62,17.12,17.14,17.36,18.10,19.13,20.28$, $21.73,22.69,23.63,25.74,25.82,26.31,32.15,34.26,36.66$, 43.01, 46.12, and 79.05.

Figure 3 describes the empirical and theoretical plots of the PDF and CDF of NFW. Figure 4 defines the P-P and Q-Q plot, and Figure 5 demonstrates the TTT plot that demonstrates the data follow a nonmonotonic hazard rate shape. Table 2 indicates MLEs, the log-likelihood, and their standard error values. Table 3 describes the goodness of fit measures; the goodness of fit measures for NFW has the smallest values that indicate that the proposed probability model has better performance than the already developed Rayleigh $(R)$, exponential $(E)$, Weibull $(W)$, Weibull exponential (W.E), and Algoharai inverse flexible Weibull (AIFW).
11.2. Data Set 2: Bank Customer Data. The data set of Aldeni et al. [20] for the waiting time of the 100 bank customers is considered with the following values:

$0.8,0.8,1.3,1.5,1.8,1.9,1.9,2.1,2.6,2.7,2.9,3.1,3.2,3.3$, $3.5,3.6,4,4.1,4.2,4.2,4.3,4.3,4.4,4.4,4.6,4.7,4.7,4.8,4.9$, $4.9,5.0,5.3,5.5,5.7,5.7,6.1,6.2,6.2,6.2,6.3,6.7,6.9,7.1,7.1$, $7.1,7.1,7.4,7.6,7.7,8,8.2,8.6,8.6,8.6,8.8,8.8,8.9,8.9,9.5$, $9.6,9.7,9.8,10.7,10.9,11.0,11.0,11.1,11.2,11.2,11.5,11.9$, $12.4,12.5,12.9,13.0,13.1,13.3,13.6,13.7,13.9,14.1,15.4$, $15.4,17.3,17.3,18.1,18.2,18.4,18.9,19.0,19.9,20.6,21.3$, 21.4, 21.9, 23, 27, 31.6, 33.1, and 38.5.

Figure 6 shows the empirical and theoretical CDF and PDF of the bank customer's data. The red line of the graph illustrates that the line is the best fitted to theoretical data as matched to other distributions. Figure 7 reveals the P-P and 


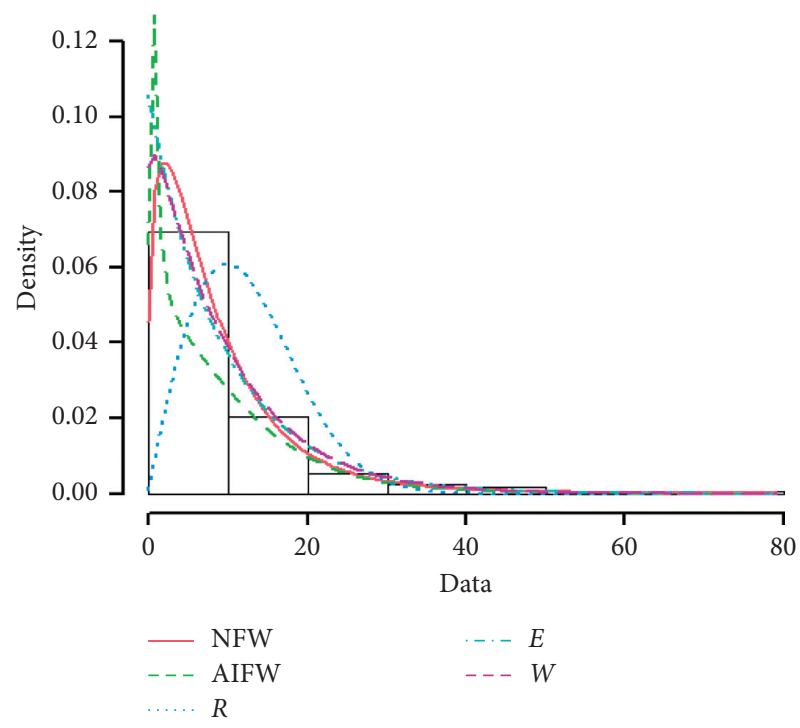

(a)

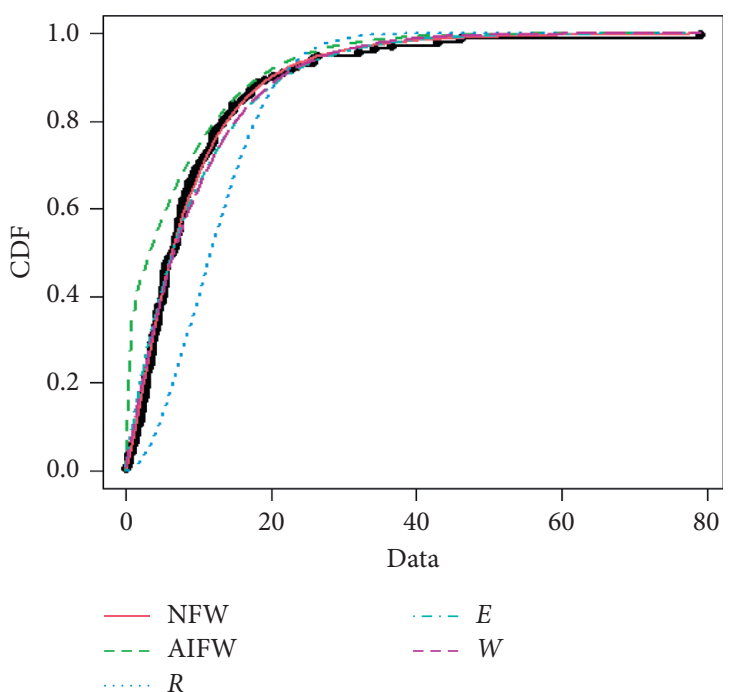

(b)

FIgURE 6: Theoretical and empirical PDF and CDF of NFW. (a) Histogram and theoretical densities. (b) Empirical and theoretical CDFs.

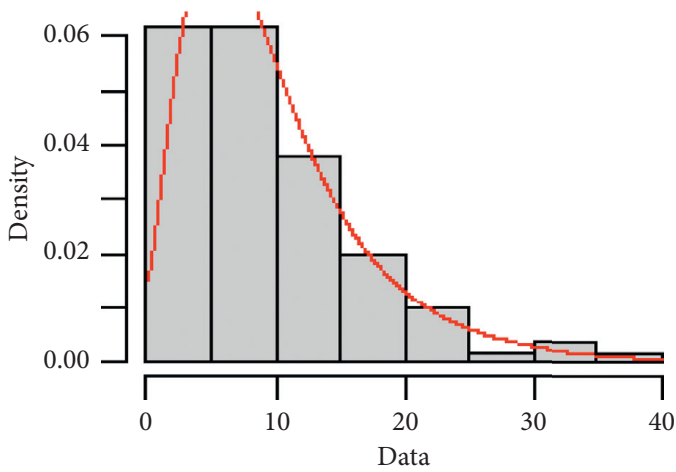

(a)

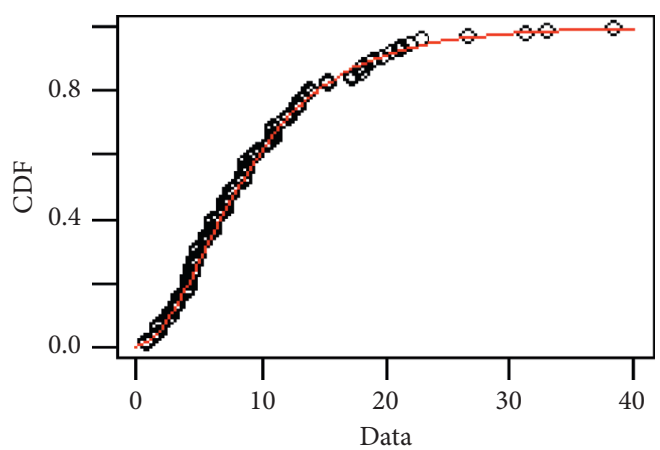

(c)

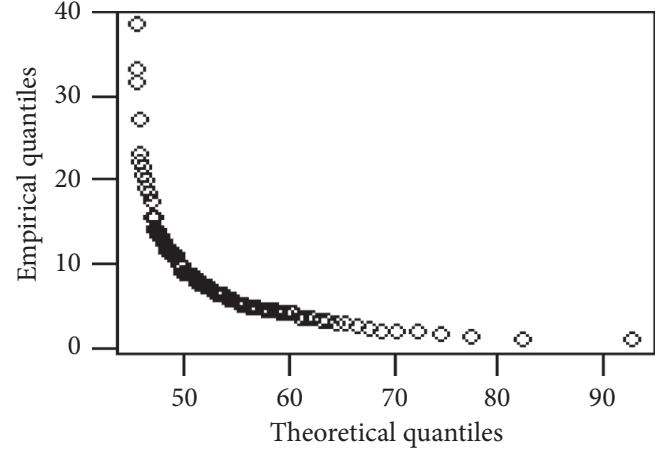

(b)

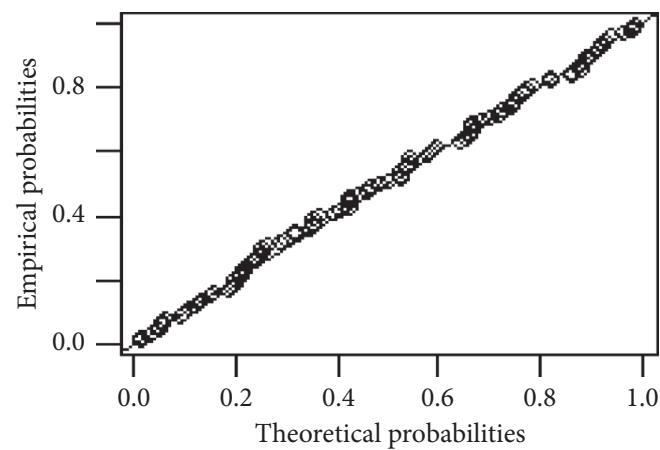

(d)

Figure 7: Theoretical and empirical PDF and CDF with Q-Q plot and P-P plot. (a) Empirical and theoretical densities. (b) Q-Q plot. (c) Empirical and theoretical CDFs. (d) P-P plot.

Q-Q plot, and Figure 8 presents the TTT plot. Figure 8 clearly demonstrates that the data fit the monotonic hazard rate structure. The MLEs are given in Table 4 including the log-likelihood and standard errors. The values in Table 5 show that the NFW performs better as compared to $R, E, W$, $W$. E, and AIFW distributions.

\section{Simulations}

To check the performance of the proposed probability model, an expression (13) was applied on the NFW distribution so that to produce artificial data. The simulations are carried out 1000 times for a various samples of size $n$ with 


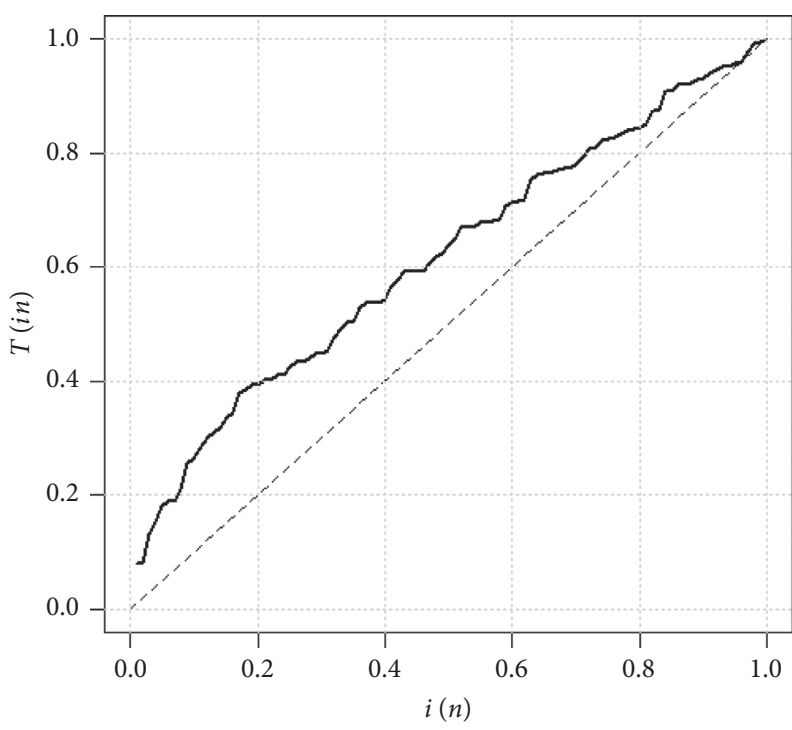

Figure 8: TTT plot of Data 2.

Table 4: Maximum likelihood estimates and their standard errors.

\begin{tabular}{lccccc}
\hline Model & $W$ & $A$ & MLE & Standard error & -Log \\
(likelihood)
\end{tabular}

TABLE 5: Goodness of fit measures of the NFW for bank customer data.

\begin{tabular}{|c|c|c|c|c|}
\hline Models & AIC & CAIC & $\mathrm{BIC}$ & HQIC \\
\hline New Flexible Weibull & 640.9464 & 641.1964 & 648.7619 & 644.1095 \\
\hline Weibull exponential & 660.0418 & 660.0826 & 662.6469 & 661.0961 \\
\hline Weibull & 660.4802 & 660.521 & 663.0853 & 661.5345 \\
\hline Exponential & 641.4893 & 641.613 & 646.6996 & 643.598 \\
\hline Rayleigh & 647.6886 & 647.9386 & 655.5041 & 650.8517 \\
\hline Algoharai inverse flexible Weibull & 665.5608 & 665.6845 & 670.7711 & 667.6695 \\
\hline
\end{tabular}

TABLE 6: Mean square errors (MSEs) and their biases.

\begin{tabular}{|c|c|c|c|c|c|c|c|}
\hline Parameters & $N$ & $\operatorname{MSE}(a)$ & MSE $(b)$ & $\operatorname{MSE}(c)$ & Bias $(a)$ & Bias $(b)$ & Bias $(c)$ \\
\hline$a=0.5$ & 30 & 37.13504 & 3.452103 & 0.002435212 & 4.724018 & 0.3978474 & 0.02786498 \\
\hline$b=5.5$ & 50 & 29.53178 & 2.42134 & 0.001603071 & 4.248159 & 0.6962165 & 0.02253221 \\
\hline$c=0.2$ & 70 & 25.98597 & 1.990059 & 0.00125555 & 4.063418 & 0.7685703 & 0.02069735 \\
\hline$a=0.5$ & 100 & 33.19275 & 0.806222 & 0.03741665 & 2.914864 & 0.1204437 & 0.0218083 \\
\hline$b=3.5$ & 150 & 15.96988 & 0.6159023 & 0.03069462 & 1.922832 & 0.05043382 & 0.01320171 \\
\hline$c=1.2$ & 200 & 11.7601 & 0.5113136 & 0.02721322 & 1.570882 & 0.04354617 & 0.01142842 \\
\hline
\end{tabular}

different sets of parameters. Table 6 provides the standard errors and their maximum likelihood estimates. The table clearly shows that as the sample size increases, both the standard errors and ML estimates decrease. The general function for calculating the bias and mean square error are given by 


$$
\begin{aligned}
& \text { MSE }=\frac{1}{W} \sum_{i=1}^{W}\left(\widehat{\alpha}_{i}-\alpha\right)^{2}, \\
& \text { Bias }=\frac{1}{W} \sum_{i=1}^{W}\left(\widehat{\alpha}_{i}-\alpha\right) .
\end{aligned}
$$

\section{Conclusion and Discussion}

The paper focuses on generating a new family of probability distributions called New Flexible Family of distributions or in short NFF. The proposed family of distribution is applied to a Weibull distribution called New Flexible Weibull (NFW) distribution. Various statistical properties including the entropy measures, order statistics, and moment generating function are determined. The parameters are estimated by using the usual method called maximum likelihood estimates. To achieve the main objectives of the paper, the proposed probability model is applied to real data sets with extreme values, where one datum follows a monotonic hazard rate and a second data set capturing the nonmonotonicity of the hazard rate function. Moreover, the significance of parameters is investigated using a simulation study and it has been shown that the parameters lead to a flexible results. Hence, it has been established that NFW produces a superior fit when modeling data sets with extreme values as opposed to the existing distributions to these data sets. A future research study should also be conducted to extend the proposed model flexibility for the extreme value data sets via transmutation techniques and some others.

\section{Data Availability}

The data sets are taken from the literature.

\section{Conflicts of Interest}

The authors have no conflicts of interest.

\section{References}

[1] M. Ijaz, S. M. Asim, M. Alamgir, M. Farooq, S. A. Khan, and S. Manzoor, "A gull alpha power weibull distribution with applications to real and simulated data," PLoS One, vol. 15, no. 6, Article ID e0233080, 2020.

[2] N. Eugene, C. Lee, and F. Famoye, "Beta-normal distribution and its applications," Communications in Statistics - Theory and Methods, vol. 31, no. 4, pp. 497-512, 2002.

[3] M. C. Jones, "Families of distributions arising from distributions of order statistics," Test, vol. 13, no. 1, pp. 1-43, 2004.

[4] K. Zografos and N. Balakrishnan, "On families of beta- and generalized gamma-generated distributions and associated inference," Statistical Methodology, vol. 6, no. 4, pp. 344-362, 2009.

[5] C. Alexander, G. M. Cordeiro, E. M. M. Ortega, and J. M. Sarabia, "Generalized beta-generated distributions," Computational Statistics \& Data Analysis, vol. 56, no. 6, pp. 1880-1897, 2012.
[6] M. Amini, S. M. T. K. MirMostafaee, and J. Ahmadi, "Loggamma-generated families of distributions," Statistics, vol. 48, no. 4, pp. 913-932, 2014.

[7] M. M. Ristić and N. Balakrishnan, "The gamma-exponentiated exponential distribution," Journal of Statistical Computation and Simulation, vol. 82, no. 8, pp. 1191-1206, 2012.

[8] H. Torabi and H. Narges Montazeri, "The gamma-uniform distribution and its applications," Kybernetika, vol. 48, no. 1, pp. 16-30, 2012.

[9] A. Alzaatreh, C. Lee, and F. Famoye, "A new method for generating families of continuous distributions," Metron, vol. 71, no. 1, pp. 63-79, 2013.

[10] G. M. Cordeiro, M. M. O. Edwin, and D. C. C. da Cunha, "The exponentiated generalized class of distributions," Journal of Data Science, vol. 11, no. 1, pp. 1-27, 2013.

[11] H. Torabi and N. H. Montazeri, "The logistic-uniform distribution and its applications," Communications in Statistics Simulation and Computation, vol. 43, no. 10, pp. 2551-2569, 2014.

[12] A. Alzaatreh, F. Famoye, and C. Lee, "The gamma-normal distribution: properties and applications," Computational Statistics and Data Analysis, vol. 69, pp. 67-80, 2014.

[13] M. H. Tahir, G. M. Cordeiro, M. Alizadeh et al., "The odd generalized exponential family of distributions with applications," Journal of Statistical Distributions and Applications, vol. 2, no. 1, pp. 1-28, 2015.

[14] M. H. Tahir, G. M. Cordeiro, A. Alzaatreh, M. Mansoor, and M. Zubair, "The logistic-X family of distributions and its applications," Communications in Statistics -Theory and Methods, vol. 45, no. 24, 2015b.

[15] G. M. Cordeiro, M. Alizadeh, and P. R. Diniz Marinho, "The type I half-logistic family of distributions," Journal of Statistical Computation and Simulation, vol. 86, no. 4, pp. 707728, 2016.

[16] A. S. Hassan and M. Elgarhy, "Kumaraswamy Weibull-generated family of distributions with applications," Advances and Applications in Statistics, vol. 48, no. 3, pp. 205-239,, 2016.

[17] M. H. Tahir, M. Zubair, M. Mansoor, M. Alizadehk, and G. G. Hamedani, "A new Weibull-G family of distributions," Hacettepe Journal of Mathematics and Statistics, vol. 45, no. 2, pp. 629-647, 2016.

[18] Z. M. Nofal, A. Z. Afify, H. M. Yousof, and G. M. Cordeiro, "The generalized transmuted-G family of distributions," Communications in Statistics-Theory and Methods, vol. 46, no. 8, pp. 4119-4136, 2017.

[19] Z. Ahmad, "A new generalized class of distributions: properties and estimation based on type-I censored samples," Annals of Data Science, vol. 7, no. 2, pp. 243-256, 2020.

[20] M. Aldeni, C. Lee, and F. Famoye, "Families of distributions arising from the quantile of generalized lambda distribution," Journal of Statistical Distributions and Applications, vol. 4, no. 1, pp. 1-18, 2017.

[21] A. Alzaghal, F. Famoye, and C. Lee, "Exponentiated TX family of distributions with some applications," International Journal of Statistics and Probability, vol. 2, no. 3, p. 31, 2013.

[22] M. Ijaz, S. M. Asim, and Alamgir, "Lomax exponential distribution with an application to real-life data," PLoS One, vol. 14, no. 12, Article ID e0225827, 2019.

[23] R. Al-Aqtash, F. Famoye, and C. Lee, "On generating a new family of distributions using the logit function," Journal of Probability and Statistical Science, vol. 13, no. 1, pp. 135-152, 2015. 
[24] G. M. Cordeiro, M. Alizadeh, T. G. Ramires, and E. M. M. Ortega, "The generalized odd half-Cauchy family of distributions: properties and applications," Communications in Statistics - Theory and Methods, vol. 46, no. 11, pp. 56855705, 2017.

[25] M. A. Nasir, M. Aljarrah, F. Jamal, and M. H. Tahir, "A new generalized Burr family of distributions based on quantile function," Journal of Statistics Applications and Probability, vol. 6, no. 3, pp. 1-14, 2017.

[26] M. E. Ghitany, F. A. Al-Awadhi, and L. A. Alkhalfan, "Marshall-olkin extended lomax distribution and its application to censored data," Communications in Statistics-Theory and Methods, vol. 36, no. 10, pp. 1855-1866, 2007.

[27] M. Ijaz, M. Asim, and A. Khalil, "Flexible lomax distribution," Songklanakarin Journal of Science and Technology, vol. 42, no. 5, pp. 1125-1134, 2019.

[28] M. Ali, A. Khalil, M. Ijaz, and N. Saeed, "Alpha-Power Exponentiated Inverse Rayleigh distribution and its applications to real and simulated data," PLoS One, vol. 16, no. 1, Article ID e0245253, 2021.

[29] A. EL-Baset, A. Ahmad, and M. G. M. Ghazal, "Exponentiated additive Weibull distribution," Reliability Engineering and System Safety, vol. 193, Article ID 106663, 2020.

[30] W. Weibull, "Wide applicability," Journal of Applied Mechanics, vol. 103, no. 730, pp. 293-297, 1951.

[31] J. M. F. Carrasco, E. M. M. Ortega, and G. M. Cordeiro, “A generalized modified Weibull distribution for lifetime modeling," Computational Statistics \& Data Analysis, vol. 53, no. 2, pp. 450-462, 2008.

[32] G. M. Cordeiro, E. M. M. Ortega, and A. J. Lemonte, "The exponential-Weibull lifetime distribution," Journal of Statistical Computation and Simulation, vol. 84, no. 12, pp. 25922606, 2014.

[33] A. Saboor, H. S. Bakouch, and M. N. Khan, "Beta sarhan-zaindin modified Weibull distribution," Applied Mathematical Modelling, vol. 40, no. 13-14, pp. 6604-6621, 2016. 